\title{
Problems of anaesthesia in the burnt child
}

\author{
GORDON H. BUSH \\ M.A., D.M., F.F.A., R.C.S., D.A. \\ Alder Hey Children's Hospital, Liverpool, 12
}

\begin{abstract}
Summary
The problems of providing satisfactory anaesthesia for the burnt child, both in the initial treatment, in repeated dressings and in the correction of subsequent deformities are discussed, and the dangers and complications of general anaesthesia emphasized.
\end{abstract}

THE treatment of burns can be conveniently divided into three phases: the initial period of resuscitation, the more prolonged stage during which the burned areas are desloughed and covered with skin grafts, and finally the protracted years when plastic surgery is required to improve the appearance and to restore function to the grafted areas.

In the initial treatment of the child, anaesthesia may be required for the cleansing and dressing of the burned area. Providing resuscitative measures have been adequate and there are no further complications, anaesthesia presents little difficulty. However, it is at this stage that respiratory complications may be most evident and are due to a variety of causes. The presence of overwhelming smoke and heat at the time of the conflagration may give rise to a period of acute hypoxia and if this is not lethal immediately, varying degrees of cerebral damage may have been produced which will be apparent subsequently, with a development of neurological signs (Warlow \& Hinton, 1969). A further potent cause of hypoxia is the inhalation of carbon monoxide with the production of carboxyhaemoglobin and the satisfactory appearance of the patient delays diagnosis and may result in the condition being completely overlooked.

The inhalation of hot and irritant gases produces important and distinct pulmonary changes. The inhalation of hot air produces serious sequelae in the tracheobronchial tree and the site of damage is dependent upon the temperature and humidity of the inspired gases. The higher the temperature and humidity the more extensive are the pathological lesions. Hot air cools rapidly when it enters the air passages producing its main effect on the upper airway and larynx, whilst steam affects the whole length of the airway because its latent heat is released when it changes to water. Inhalation of irritant gases, particularly sulphurous and nitrous fumes, is particularly dangerous and lethal to experimental animals and the recent experiences with contaminated nitrous oxide suggest that nitrous and sulphurous compounds are equally lethal in man affecting principally the lung parenchyma.

\section{Pulmonary complications}

Pulmonary complications following the inhalation of hot air and fumes include:

\section{Laryngeal oedema}

This principally affects the supraglottic area and traces of carbon are often to be seen adhering to those structures on laryngoscopy. Should upper airway obstruction be very severe an artificial airway or tracheostomy may be necessary. In any event an atmosphere of high humidity and oxygen are required. Characteristically, in these patients with this condition the burned area is usually quite small since they are the victims of a smouldering fire of small heat output and excess smoke.

\section{Laryngotracheitis}

Excess heat in the upper trachea results in the destruction of the cilia and epithelium of the upper trachea which heals by the formation of a pseudo-membrane covering the ulcerated area. This membrane is composed of a fibrous exudate and will undergo separation and then curl up in the trachea or become detached, producing complete respiratory obstruction. These patients therefore require the closest observation and repeated laryngoscopy and bronchoscopy may be necessary to remove the detached portion of the membrane.

\section{Pulmonary oedema}

Acute parenchymal congestion with areas of haemorrhage and oedema follows the inhalation of nitrous and sulphurous compounds. A raised inspired oxygen concentration and controlled pulmonary ventilation may be necessary to tide the patient over this period of pulmonary injury. 
Acute respiratory symptoms may also occur due to other causes, in direct involvement of the airway associated with burns around the face and neck, particularly if the lesion is caused by an electric burn, which produces a deep area of tissue destruction with considerable local oedema.

Respiratory failure with an absent cough reflex and accumulation of secretions is particularly liable to follow cerebral damage with convulsions and coma consequent upon hypoxia occurring at the time of the accident or following the application of hexylene glycol (Procter, 1967).

\section{Dangers of general anaesthesia}

A circumferential burn of the chest with eschar formation may produce considerable limitation of respiratory excursion and predispose to atelectasis and subsequent infection. Any serious degree of respiratory impairment should be relieved by the incision of the eschar. The majority of children who present for desloughing or grafting of burned areas do not present any difficulty to the anaesthetist. However, when large areas of skin are involved the presence of infection, toxaemia, inanition, anaemia and metabolic disturbances considerably increase the risk of anaesthesia. Despite these hazards, however, anaesthesia must be provided on frequent occasions since in extensive burns satisfactory conditions can only be obtained for the patient and the surgeon by general anaesthesia. Local analgesia is impracticable because children are unsuitable subjects for this technique and the areas involved either in loss due to burning or that required for grafting are too extensive. Hypnosis can provide excellent conditions and other advantages associated with the posthypnotic state but unfortunately does not have universal application.

For minor procedures such as change of dressing, it is often argued that repeated general anaesthesia is so hazardous as to be contra-indicated particularly in view of the disturbances in nutritional requirements at this time of excessive catabolism. The alternatives to general anaesthesia would seem to be either basal narcosis using heavy doses of either rectal pentothal, neuroleptic drugs or dissociative anaesthesia with Ketamine, which if successful must produce disadvantages akin to that of some states of general anaesthesia or the use of an inhalational analgesic mixture. Unfortunately a number of children will not co-operate sufficiently to make inhalational analgesia a satisfactory method for minor procedures in all cases. For all but the most minor dressing a short general anaesthetic from which recovery is rapid would seem to be far preferable and providing heavy premedication is avoided, little interference with nutrition will occur.

General anaesthesia for desloughing and grafting should aim to provide a pleasant pre-operative period, a tranquil induction, utilize minimal toxic agents, allow rapid awakening postoperatively without restlessness and have a low incidence of vomiting and other sequelae. Endotracheal intubation must be performed in the majority of cases to ensure a satisfactory airway either because of the location of the burned area or to provide access to the donor areas, and when carefully performed carries no additional risk. These criteria may be satisfied by the use of oral trimeprazine tartrate given $4 \mathrm{hr}$ before induction followed by intramuscular morphine and atropine proportional to body weight. Anaesthesia is induced with intravenous thiopentone given through a 27 gauge needle and maintained with $70 \%$ nitrous oxide in oxygen using tubucurarine to achieve full control of pulmonary ventilation. Before the termination of the procedure, pethidine $1 \mathrm{mg} / \mathrm{kg}$ body weight is given intravenously to provide analgesia for the donor areas, and the curarization is reversed with a standard dose of atropine and prostigmin.

A reliable intravenous infusion is essential to enable blood replacement to keep pace with the large volume lost during these procedures. A cutdown infusion is required only in exceptional circumstances as the peripheral veins are easily cannulated percutaneously, a procedure facilitated by the vasodilatation following the induction of general anaesthesia and by the concomitant wasting of the subcutaneous tissues. Massive blood transfusions are required for these procedures and on occasions a total blood replacement may be given. Considerable difficulties arise in estimating the blood requirements, since blood pressure monitoring may be impossible and reliance has to be placed upon a number of clinical signs, not the least being the state of the peripheral pulse, the pulse rate and peripheral perfusion. Any estimate of blood loss by gravimetric or colorimetric methods under-records the actual loss by about $50 \%$.

The greatest danger of massive blood transfusion would appear to be the tendency for selective cardiac cooling to occur. Oesophageal temperatures have been known to fall from $37^{\circ}$ to $31.5^{\circ} \mathrm{C}$ in the course of $3 \mathrm{hr}$ during an infusion of blood amounting to $75 \%$ of the child's blood volume. A blood warming coil immersed in a thermostatically controlled water bath through which the blood may run will be advisable in all these cases. Evidence continues to mount concerning the disastrous consequences of administering suxamethonium to these patients. Instability of certain membranes and the excessive leakage of potassium following suxamethonium leads to dangerously high levels of serum potassium which may result in ventricular fibrillation and circulatory arrest. Fortunately, the condition is self- 
limiting and providing circulation is continued the potassium level falls and cardiac action is restored. It is for this reason that suxamethonium should have no place in anaesthesia for the burned patient. The resistance to tubocurarine that is a feature of burned patients is almost certainly due to the increase in gamma-globulin as a result of the presence of infection and toxaemia. Since the binding of pancuronium is apparently not associated with any particular plasma protein fraction marked resistance to pancuronium should not develop in the burned patient.

Even following the healing of the burned areas, the badly scarred child still requires further surgery to relieve contractions or to improve the cosmetic appearances. Particular difficulties present when there are severe contractions around the head and neck. Not only may the neck be severely contracted but the mouth and nose may also be scarred making initial intubation or even the insertion of an oral airway impossible. Anaesthesia may be induced with a sleep dose of thiopentone and maintained with halothane and oxygen. At this stage a nasopharyngeal tube may be invaluable in holding the base of the tongue away from the pharyngeal wall. The surgeon may then incise the contracture of the neck and when the head is extended, intubation under direct vision can be performed. An alternative method involves the division of the scar contractures under local analgesia followed by general anaesthesia.

Apart from all else, an humane, friendly, gentle and encouraging approach is essential when dealing with the burnt child. The burns of today are the suffering of tomorrow and the scars of a lifetime.

\section{References}

Procter, D.S.C. (1966) Coma in burns; the cause traced to dressings. South African Medical Journal, 40, 1116.

WaRlow, C.P. \& HinTon, P. (1969) Early neurological disturbances following relatively minor burns in children. Lancet, ii, 978.

\section{Discussion}

DR Russell M. Davies: Thank you very much indeed, Dr Bush, for a fascinating talk. There are three pointsone question and possibly two comments. The first question is: for the general anaesthetic which you give, your premedication starts $4 \mathrm{hr}$ before the intended time of surgery. Is there any feeding between that point and the postoperative period? If not, I would question the thesis whether this is not a good thing. The work (particularly of Sutherland) very well documents that the food intake which is lost in a burnt patient until at least he has got skin cover is never recovered if there is a period of starvation for any reason.

The second point is purely a statement of the question of blood loss. We became extremely concerned with this some 3 years ago particularly in relation to children where the guesses came even further away from the truth than one hoped for. Of course, acting on one's guesses, the damage that one can do became correspondingly greater. So we spent a year looking for a method of measuring blood loss, particularly in desloughing operations, and this is now done as routine in all desloughing operations at all times. The method we use employs a modification of a machine made in Sweden called a Perometer which is really a haemoglobin measurement technique. All the swabs and washings and so forth are put in the washing machine which has a continual circulation of the lysed fluid. The measurement is essentially a spectroscopic method, and this we found gives us a remarkably high degree of accuracy.

Our justification for saying that it is accurate is this. If one assumes that the circulating volume of a child is of the order of $40 \mathrm{ml} / \mathrm{lb}$, and taking an immediate preoperative haemoglobin, one can make a reasonable estimate of the amount of haemoglobin which is required for bringing the child's haemoglobin to a particular level. This can be calculated in terms of the volume of blood. If to this is added the volume of blood which is lost during the surgery, and the Perometer provides an accuracy of plus or minus $3 \%$, this leaves us with the postoperative loss into the dressings, particularly the donor site but to a lesser extent to the recipient site. We have found that again if we remove the dressings even as late as $\mathbf{1 0}$ days after the operative incident and put them through the washing machine with a lysing fluid one can make a reasonable estimate of the postoperative loss. This again is an estimate.

So we now have three fractions-the pre-operative deficit of haemoglobin, the blood loss during surgery, and thirdly the loss into the dressings postoperatively. We have found that we can make a reasonable guessand it is a guess-as to how much is lost $/ \mathrm{m}^{2}$ of deslough.

The reason for saying that we have been reasonably accurate is this. Starting with the pre-operative haemoglobin, say $65 \%$, we then take these three factors and say that we would like to finish up with a postoperative haemoglobin $48 \mathrm{hr}$ postoperatively of $95 \%$; and perhaps some of the surgeons here would support me or shoot me down, but more often than not we are within $5 \%$ of the predicted level. Particularly with children this removes a lot of the worry of how much blood to give. I think even more important, it has given us the opportunity of saying when we will not transfuse, particularly, a child. A child will start with a high haemoglobin. The measured loss is small; the desloughed site is small. So we then have the courage not to transfuse a patient, because $I$ am sure we would all agree that transfusion in itself carries a morbidity factor.

This, in our hands, has proved a worthwhile tool to explore still further, and we use it now as a routine.

The third point-and this is applying geometry-deals with the last two slides of the chin tied down on the chest. I would agree absolutely-I am sure we all would-with 
the method you used for anaesthetizing these children. If we could imagine the retroglossal space in the pharynx, there must be an asymmetric pyramidal space above the chords bounded posteriorly by the posterior pharynx and anteriorly by the posterior base of the tongue. In fact, we had a small child from Vietnam who presented this picture to the $n$th power. We did soft tissue X-rays and measured this pyramidal space, and in this child this measured half an inch antero-posteriorly and three quarters of an inch up and down. We did exactly what you suggested, banking on the validity of the $\mathrm{X}$-rays and manipulating the tube until it lay in that space, and asked the surgeon to continue. We should be assured to a certain extent that there is this pyramidal space, which is almost impossible to obliterate even in the grossly deformed child.

DR BUSH: As regards feeding, I accept what you say. It is certainly my impression-I have no factual evidence - that our children now do not lose weight as much as they did 5-10 years ago. This was despite apparently the same routine of anaesthesia. I am certain that the recent advances that have been made in the local treatment of burns have prevented the degree of infection that we used to see. One no longer sees the extent of infection, where one used to take off dressings which were swimming in pus. I think that this reduction in toxaemia-infection has a lot more to do with maintaining the nutrition of these people than the period of starvation that we have to give them. I accept that.

As far as the Perometer is concerned, we have been using this. Ours broke down in January and is apparently now beyond repair due to the use it has had. So we do not use it; but we have always used a similar method, a colorimetric method. But we found in any case-and it was shown, I think, by Thornton from Sheffield-that this method gives you only a $66 \%$ actual loss when compared with blood volume measurement. Assuming that one gets a complete extraction, this is what is found, but in our case the amount of extraction because of the heavily contaminated swabs is much less than ideal, and that is why I say that a $50 \%$ estimate is reasonable. That is all entirely guesswork because one does not know the haemoglobin in the blood that you are given. It depends on how old it is, and so forth.

As far as the postoperative infusion is concerned, if we do a $20 \%$ grafting we treat these patients as a $20 \%$ burn for 8-12 hr postoperatively, and we infuse plasma to take the place of the plasma losses that will occur during this immediate postoperative period. But I agree that it is extremely difficult and it is entirely an estimate.

DR Hinton: May I mention one factor which has to be borne in mind, whether one is trying to consider the accuracy of one's measurements of blood losses or replacing them. Neither the blood loss nor the blood volume measurements may be entirely accurate, but there is the fact that the normal blood volume of a healthy subject is not necessarily the optimal blood volume of a very ill patient, particularly the septicaemic patient. A very septicaemic patient needs more blood than he has lost-not exactly the same amount.

DR W. N. Rollason: One often finds the central venous pressure a useful monitor. Does Dr Bush use this?

DR BUSH: No, not at all, largely because they often require three or four desloughings, and it is difficult-

DR Rollason: I was thinking of the initial resuscitation, when you can get a needle into a vein in the burnt area.

DR Bush: No. For initial resuscitation we go entirely on the volume of urine output/hr and the clinical condition of the patient.

DR ANTIC (Belgrade): I would like to ask about hypnosis. Have you used hypnosis with children, and if so what have been your results? Up to 1967 , out of 5000 cases of hypnosis we had 841 cases with sufficiently good results. Only 123 failed. We prefer to use hypnosis when possible when you have a properly trained man and if you have enough time to do it, instead of ketamine anaesthesia. I would like to know your view.

DR BUSH: I can answer the first part of your question extremely quickly. I have no experience. I think it would be extremely good, as you say, if one has got the time and personnel to do it. As far as ketamine anaesthesia is concerned, I should like to leave it until that subject comes up for discussion. 